\title{
Yttrium-90 Microsphere Radioembolization for Treatment of Lung Cancer Hepatic Metastases
}

\author{
Ron C. Gaba Janesh Lakhoo \\ Department of Radiology, University of Illinois Hospital and Health Sciences \\ System, Chicago, III., USA
}

\section{Key Words}

Lung cancer $\cdot$ Liver metastases $\cdot$ Yttrium-90 radioembolization

\begin{abstract}
Because stage 4 lung cancer is associated with dismal 5-year survival rates, new treatment approaches targeting extrapulmonary disease are necessary. Yttrium-90 microsphere radioembolization is an emerging treatment for metastatic hepatic malignancies that results in high tumor response rates and extended patient survival. To date, application of this therapy toward management of lung cancer hepatic metastases has not been extensively described. Herein, we present 2 cases of effective yttrium-90 radioembolization for treatment of lung cancer hepatic metastases, and emphasize the potential coadjuvant value of this procedure in patients with advanced-stage lung cancer and liver-dominant metastatic disease.
\end{abstract}

\section{Introduction}

Yttrium-90 ( $\left.{ }^{90} \mathrm{Y}\right)$ microsphere radioembolization is an emerging treatment for metastatic hepatic malignancies [1]. In this procedure, 20-60 micron-sized $\beta$-radiationemitting particles are administered to liver tumors using a transcatheter approach to deliver high-dose localized internal radiation [2]. This therapy takes advantage of the predominant hepatic arterial flow to liver tumors; cancerous tissue derives $80-100 \%$ of its blood supply from the hepatic arterial system [3], while normal liver gains 75$80 \%$ of its perfusion from the portal vein. Radioactive microspheres delivered from a catheter in the hepatic artery are preferentially deposited in malignant tissue at a 3:1 to 20:1 ratio compared to normal liver [4], and result in high tumor response rates and extended survival [5]. While the use of ${ }^{90} \mathrm{Y}$ radioembolization for treatment of hepatic 
metastases from colorectal [6], neuroendocrine [7], and breast cancer [8] has been broadly investigated, application of this therapy toward management of lung cancer hepatic metastases has not been extensively documented [9]. Herein, we present 2 cases of effective ${ }^{90} \mathrm{Y}$ radioembolization for treatment of chemorefractory lung cancer hepatic metastases, and highlight the potential coadjuvant value of this procedure in patients with stage 4 lung cancer and liver-dominant metastatic disease.

\section{Case Reports}

Institutional review board approval is not required for small retro spective case studies at the authors' hospital.

Case 1

A 68-year-old woman with a 50-pack-year smoking history presented with a complaint of dyspnea and cough. She underwent a chest radiograph followed by computed tomography (CT), which revealed a $4.8 \times 2.8 \mathrm{~cm}$ mass in the upper lobe of the left lung (fig. 1a). A bronchoscopy was performed 3 months after presentation for tissue diagnosis, but both bronchoalveolar lavage and transbronchial needle aspirate were negative for malignancy. The patient was then referred to Interventional Radiology (IR) for CT-guided transthoracic biopsy, which yielded a diagnosis of welldifferentiated squamous cell carcinoma. Work-up with whole-body positron emission tomography (PET) and brain magnetic resonance imaging demonstrated stage 3b (T4N2M0) disease. As such, the patient was not a candidate for surgical resection, and was placed on chemoradiation treatment.

The patient began chemoradiation 5 months after initial presentation. She was given cisplatin and etoposide for 1 cycle, and was treated with a 66-Gy radiation dose using 2-field 3-dimensional conformal radiotherapy. The patient was subsequently followed with CT imaging; a scan performed 2 months after therapy showed size reduction of the primary tumor to $4.6 \times 2.2 \mathrm{~cm}$, but also demonstrated a new $2.0 \times 1.9 \mathrm{~cm}$ lesion in the right hepatic lobe suspicious for metastasis. She was started on second-line chemotherapy 2 months later with carboplatin AUC6 and paclitaxel. Follow-up CT 4 months later showed smaller size of the lung mass (fig. 1a), but increased size of the hepatic metastasis to $4.3 \times 4.3 \mathrm{~cm}$ (fig. $1 \mathrm{~b}$ ). The patient was therefore started on third-line chemotherapy with gemcitabine for 4 cycles. CT scan 2 months after completing chemotherapy showed no decrease in the size of the hepatic metastasis and revealed a new $0.9 \mathrm{~cm}$ satellite lesion.

At this point, the patient was referred to IR for transcatheter treatment of her lung cancer hepatic metastases. Given good baseline performance status, with Eastern Cooperative Oncology Group (ECOG) status 1, as well as preserved liver function, the patient was a good candidate for ${ }^{90} \mathrm{Y}$ microsphere therapy. In preparation for treatment, the patient underwent mapping hepatic arteriogram with technetium-99m macroaggregated albumin (99mTc-MAA) scan for lung shunt fraction (LSF) calculation. A conventional common femoral artery approach was used. Right hepatic arteriogram demonstrated a large hypervascular right hepatic lobe tumor (fig. 1c), and the hepatic vascular anatomy was found to be amenable for ${ }^{90} \mathrm{Y}$ therapy. $5.4 \mathrm{mCi}$ of $99 \mathrm{mTc}-\mathrm{MAA}$ was administered from the right hepatic artery for LSF analysis. Finally, the femoral vascular access was removed and hemostasis was accomplished by manual compression. $99 \mathrm{~m}$ Tc-MAA scan demonstrated a hepatopulmonary shunt fraction of $17.7 \%$, below the $20 \%$ threshold for safe treatment [10]. The radioembolization procedure was scheduled for 1 week later.

On the day of the treatment procedure, a 5-French catheter and coaxial 3-French microcatheter were advanced into the right hepatic artery. An approximately 120-Gy radiation dose was administered via injection of $3 \mathrm{GBq}$ of ${ }^{90}$ Y-labeled glass microspheres (TheraSphere; MDS Nordion, Ottawa, Ont., Canada). Completion angiography was performed, all vascular devices were removed, and access site hemostasis was achieved by manual compression.

After an uneventful post-procedure recovery, the patient was seen 1 month later. CT scan at that time demonstrated complete necrosis of the right hepatic lesion (fig. 1d), and PET CT scan performed 2 months after treatment showed no evidence for residual viable tumor (fig. 1d). At present, the patient is alive with persistent complete response in the treated liver lesion 28 months after initial diagnosis, 21 months after progression to stage 4 disease, and 11 months after ${ }^{90}{ }^{9}$ therapy. Of note, 
she has since undergone 2 additional successful hepatic radioembolization procedures to treat new small metastases that developed since the time of initial liver therapy.

Case 2

A 53-year-old woman with a history of stage 1b (T2N0M0) left lung squamous cell carcinoma status post lobectomy and adjuvant chemotherapy with carboplatin and paclitaxel 2 years prior presented for evaluation of a solid mass on the left tongue undersurface. Biopsy demonstrated poorly differentiated squamous cell carcinoma, and the patient underwent wide local excision of the floor of mouth and left tongue, bilateral neck dissection, and flap reconstruction. One month after surgery, the patient was placed on cisplatin chemoradiation therapy during which time she received a total dose of 66 Gy. Upon completion of adjuvant therapy, she subsequently underwent PET CT imaging surveillance, and 11 months later was found to have a new $2.8 \times 2.4 \mathrm{~cm}$ left hepatic lobe mass. Ultrasound-guided biopsy was positive for metastatic squamous cell carcinoma consistent with the patient's lung primary tumor. The patient was placed on a second round of chemotherapy using cisplatin, 5-fluorouracil, and cetuximab. She was also referred to IR for concomitant transcatheter treatment of her lung cancer hepatic metastasis; given good performance status (ECOG 0) and preserved liver function, ${ }^{90} \mathrm{Y}$ radioembolization was selected.

The patient underwent mapping arteriogram in preparation for ${ }^{90} \mathrm{Y}$ radioembolization, performed from a conventional femoral artery approach. Left hepatic artery arteriogram demonstrated a hypervascular left hepatic lobe tumor ( $\underline{\text { fig. } 2}$ b), and the hepatic vascular anatomy was found to be

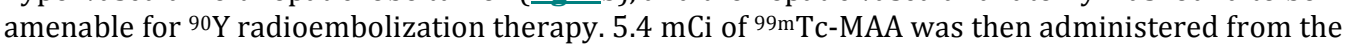
proper hepatic artery for LSF analysis. Finally, the femoral vascular access was removed and hemostasis was accomplished by manual compression. ${ }^{99 \mathrm{~m} T c-M A A}$ scan demonstrated a hepatopulmonary shunt fraction of 5.3\%. The radioembolization procedure was scheduled for 1 month later.

On the day of the treatment procedure, a 5-French catheter and coaxial 3-French microcatheter were advanced into the left hepatic artery. An approximately 120-Gy radiation dose was administered via injection of $1.57 \mathrm{GBq}$ of ${ }^{90}$ Y-labeled glass microspheres (TheraSphere; MDS Nordion). Completion angiography was performed, all vascular devices were removed, and access site hemostasis was achieved by manual compression. Following ${ }^{90} \mathrm{Y}$ administration, correct tumor targeting was confirmed by bremsstrahlung imaging (fig. $2 \mathrm{~b}$ ).

The patient experienced an uneventful post-procedure recovery and is currently continuing with her 6-cycle chemotherapy regimen. PET CT performed 2 months after ${ }^{90} \mathrm{Y}$ treatment showed complete tumor response without evidence for residual tumor (fig. 2c). At present, the patient is alive without active disease 16 months after initial diagnosis, 4 months after progression to stage 4 disease, and 2 months after ${ }^{90} \mathrm{Y}$ radioembolization therapy.

\section{Discussion}

Lung cancer is the most common malignancy worldwide and a significant cause of global morbidity and mortality. An estimated 1,600,000 new cases and 1,380,000 deaths occurred internationally in 2008 [11], and a projected 226,000 new cases and 160,000 deaths are expected in the United States in 2012 [12]. Liver metastases complicate approximately $6 \%$ of lung cancer cases at the time of initial diagnosis [13], and hepatic tumor spread occurs in up to $36 \%$ of patients over the course of their disease [14]. Unfortunately, stage 4 lung cancer is associated with $<10 \% 5$-year survival rates [15], and is most commonly treated with palliative systemic chemotherapy. New treatment approaches for this challenging disease stage are necessary to improve discouraging survival outcomes, and a role for targeted transcatheter therapy in the treatment of lung cancer liver metastases has been recently suggested in small clinical studies $[9,16]$. 
Radioembolization is a minimally invasive targeted therapy that selectively delivers internal $\beta$-radiation via the arteries that supply tumors [2]. The term 'radioembolization' describes the mode of action of this procedure, in that embolic particles act as a vehicle for delivery and administration of radiation [2]. ${ }^{90} \mathrm{Y}$ is a pure $\beta$ emitting isotope with a half-life of $64.2 \mathrm{~h}$, after which it decays into stable zirconium. The average energy of $\beta$-emission is $0.9367 \mathrm{MeV}$, and the mean tissue penetration is 2.5 $\mathrm{mm}$. One $\mathrm{GBq}\left(27 \mathrm{mCi}\right.$ ) of ${ }^{90} \mathrm{Y}$ provides a $50-\mathrm{Gy}$ dose to $1 \mathrm{~kg}$ of liver tissue; 120 -Gy tumoricidal doses are typically administered during treatment procedures. Glass (TheraSphere; MDS Nordion) and resin (SIR-Sphere; Sirtex Medical, Lane Cove, N.S.W., Australia) particles are commercially available for clinical use in the United States. Because ${ }^{90} \mathrm{Y}$ radioembolization targets tumors through a transarterial route, a planning angiographic study is required to assess visceral anatomy, identify anatomic variants, isolate the hepatic circulation, and determine tumor location for appropriate catheter placement [2]. Both the planning and treatment procedures may be performed on an outpatient basis.

In the present case series, we effectively devitalized 2 large lung cancer metastatic nodules using ${ }^{90} Y$ radioembolization. In both cases, the liver nodules represented the dominant site of active disease and were refractory to systemic chemotherapy, showing enlargement or viability despite standard of care pharmacologic treatment. Both patients showed no PET active disease in the index lesion at 10 and 2 months after treatment, indicating robust and durable complete response to therapy and significant tumor cytoreduction using the targeted treatment approach. While patients in each case were administered a treatment dose of $120 \mathrm{~Gy}$, tumor hypervascularity and preferential blood flow dictates that each lesion likely received a several thousand Gy dose [17], far exceeding 120-Gy tumoricidal levels, while normal liver parenchyma contracted low doses approximating 10-20 Gy, minimizing risk for radiation hepatitis. In this manner, internal ${ }^{90} \mathrm{Y}$ radiotherapy allows for higher cytotoxic radiation doses than traditional external beam therapy by making use of internal targeted delivery.

To date, the application of ${ }^{90} \mathrm{Y}$ radioembolization therapy toward treatment of lung cancer hepatic metastases has been specifically described in one small clinical series [9]. In 2008, Murthy et al. [9] reported on 6 patients with liver-dominant lung cancer spread who underwent ${ }^{90} Y$ treatment. Disease control was achieved in 3 of $6(50 \%)$ patients, while 3 of $6(50 \%)$ patients developed progressive disease. Median survival was 2.7 (range 1-26) months. These suboptimal outcomes may be explained on the basis of advanced disease among treated patients, of which two-thirds had multifocal bilobar liver metastases at the time of ${ }^{90} \mathrm{Y}$ therapy. In contrast, our patients were treated earlier in the disease course in the setting of solitary liver metastases that could be specifically targeted with an extraordinarily high radiation dose. Our encouraging outcomes suggest need for further investigations to assess the role of early ${ }^{90} \mathrm{Y}$ radioembolization in combination with systemic chemotherapy for lung cancer liver metastases; this treatment approach has precedent in similar ongoing trials for cancers such as metastatic colorectal carcinoma [18].

The effect of non-target pulmonary radiation is an important consideration in patients with lung cancer liver metastases who undergo ${ }^{90} \mathrm{Y}$ radioembolization, particularly those with a history of lobectomy or prior radiation, in order to avoid untoward effects on pulmonary function. Current standard of care with ${ }^{90} \mathrm{Y}$ treatment requires performance of a ${ }^{99 \mathrm{mTC}-\mathrm{MAA}} \mathrm{scan}$ in order to calculate a LSF prior to 
radioembolization; non-target pulmonary deposition of microspheres may occur due to systemic passage of particles via small intrahepatic peri-tumoral arteriovenous communications [19]. While the incidence of radiation pneumonitis is well below $1 \%$ if standard dosimetry models are used $[20,21]$, single-session doses do not exceed $30 \mathrm{~Gy}$, and total doses do not surpass $50 \mathrm{~Gy}$, high LSFs resulting in greater lung doses may necessitate ${ }^{90} \mathrm{Y}$ dose reduction to avoid radiation pneumonitis. The calculated lung doses in our patients were 21 and $4 \mathrm{~Gy}$, respectively. For comparison, patients receiving external beam radiation treatment for lung cancer are usually given approximately 60-Gy doses, but the lung volume irradiated with $>20$ Gy is minimized to decrease risk of pneumonitis [22].

In summary, we describe 2 cases of lung cancer liver metastases successfully treated with ${ }^{90} \mathrm{Y}$ radioembolization. In both cases, tumors were effectively devitalized and both patients showed excellent clinical outcome. While our clinical observations are made on the basis of a small sample size and should not be overstated, the promising results herein affirm the utility of ${ }^{90} \mathrm{Y}$ radioembolization for targeted treatment of liver metastases, and underscore the need for future investigation into such treatment strategies for advanced lung cancer.

\section{Disclosure Statement}

The authors declare that they have no conflicts of interest. 

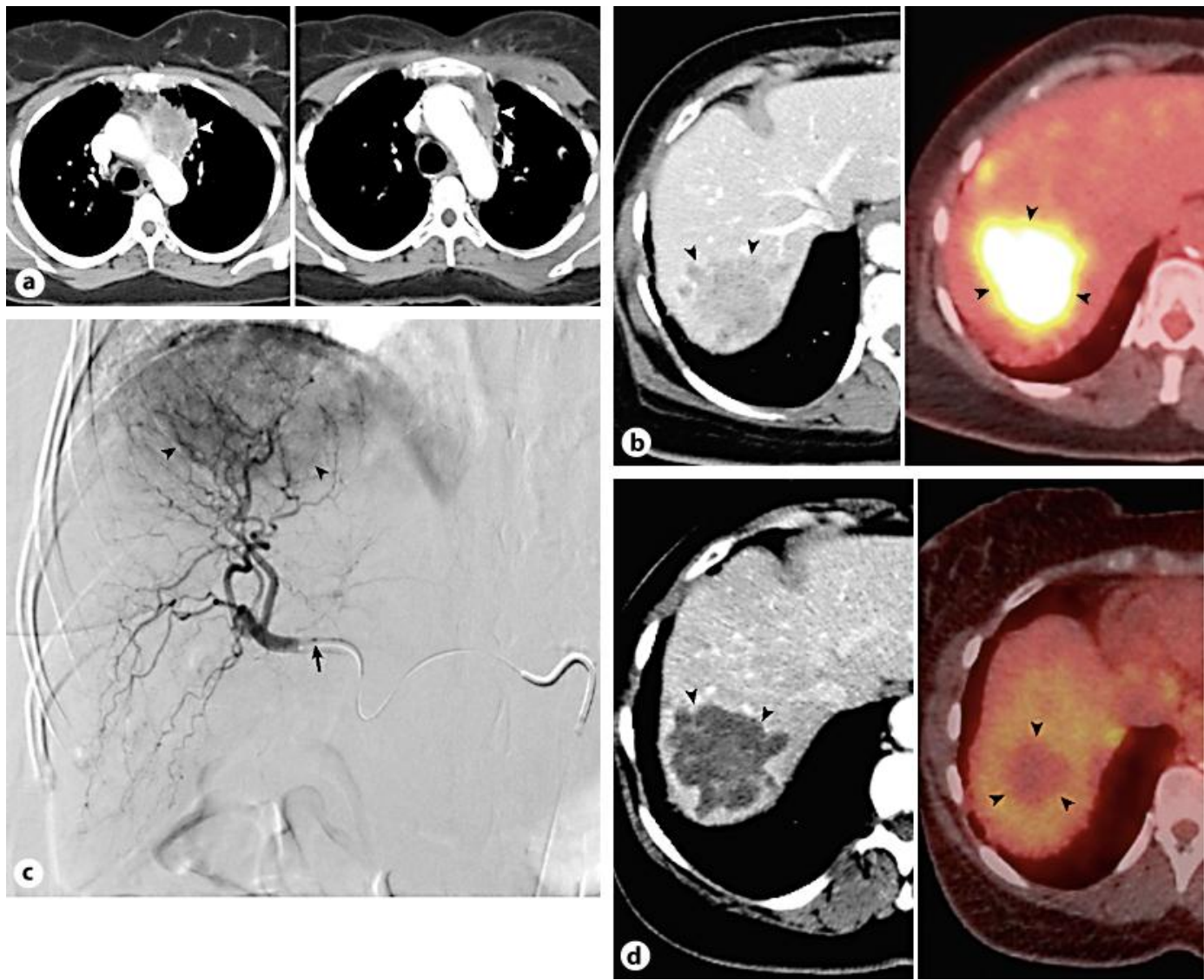

Fig. 1. Baseline contrast-enhanced CT scan (left) reveals large left upper lobe lung mass (arrowhead). Post-chemotherapy CT scan (right) demonstrates significant tumor size reduction (arrowhead) (a). CT (left) and PET CT (right) images show large hypermetabolic liver metastasis (arrowheads) (b). Digital subtraction right hepatic arteriogram displays hypervascular metastasis (arrowheads); microcatheter tip denoted by arrow (c). CT (left) and PET CT (right) images after 90Y liver therapy reveal complete tumor response (arrowheads), evidenced by CT attenuation reduction indicating necrosis, as well as complete loss of PET avidity indicating lack of viable tumor (d). 

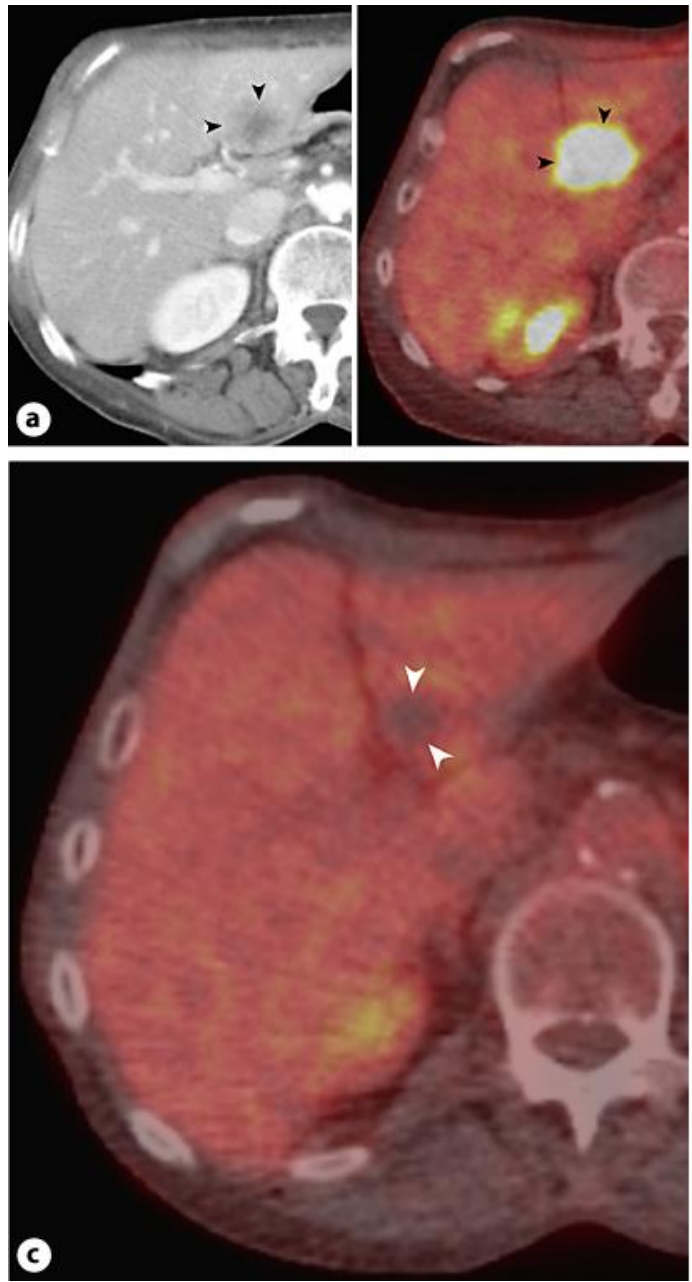

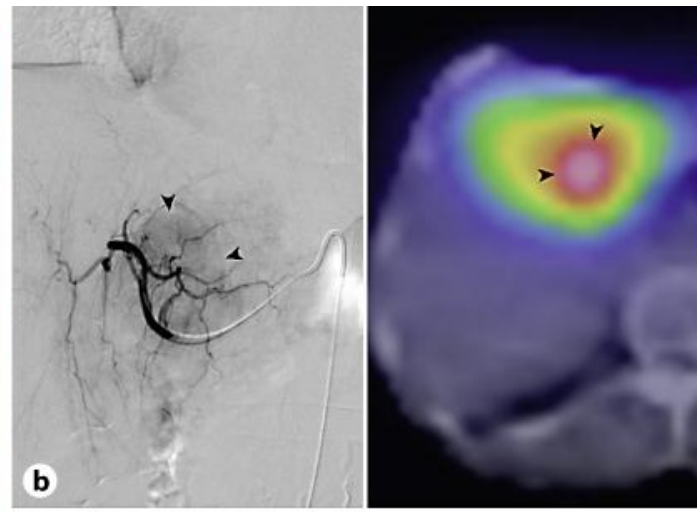

Fig. 2. Contrast-enhanced CT (left) and PET CT (right) images show hypermetabolic liver metastasis (arrowheads) (a). Digital subtraction left hepatic arteriogram (left) displays hypervascular metastasis (arrowheads); bremsstrahlung scan (right) performed after radioembolization demonstrates targeted tumor uptake of $90 \mathrm{Y}$ (b). PET CT image after $90 \mathrm{Y}$ liver therapy reveals complete tumor response (arrowheads), evidenced by complete loss of PET avidity (c).

\section{References}

1 Memon K, Lewandowski RJ, Kulik L, Riaz A, Mulcahy MF, Salem R: Radioembolization for primary and metastatic liver cancer. Semin Radiat Oncol 2011;21:294-302.

$>2$ Salem R, Thurston KG: Radioembolization with 90Yttrium microspheres: a state-of-the-art brachytherapy treatment for primary and secondary liver malignancies. Part 1: technical and methodologic considerations. J Vasc Interv Radiol 2006;17:1251-1278.

3 Breedis C, Young G: The blood supply of neoplasms in the liver. Am J Pathol 1954;30:969-977.

$\checkmark 4$ Kennedy AS, Nutting C, Coldwell D, Gaiser J, Drachenberg C: Pathologic response and microdosimetry of (90)Y microspheres in man: review of four explanted whole livers. Int J Radiat Oncol Biol Phys 2004;60:1552-1563.

-5 Salem R, Thurston KG: Radioembolization with yttrium-90 microspheres: a state-of-the-art brachytherapy treatment for primary and secondary liver malignancies: part 3: comprehensive literature review and future direction. J Vasc Interv Radiol 2006;17:1571-1593. 
6 Mulcahy MF, Lewandowski RJ, Ibrahim SM, et al: Radioembolization of colorectal hepatic metastases using yttrium-90 microspheres. Cancer 2009;115:1849-1858.

-7 Kennedy AS, Dezarn WA, McNeillie P, et al: Radioembolization for unresectable neuroendocrine hepatic metastases using resin 90Y-microspheres: early results in 148 patients. Am J Clin Oncol 2008;31:271279.

8 Jakobs TF, Hoffmann RT, Fischer T, et al: Radioembolization in patients with hepatic metastases from breast cancer. J Vasc Interv Radiol 2008;19:683-690.

9 Murthy R, Mutha P, Lee JH, Oh Y: Yttrium-90-labeled microsphere radioembolotherapy of liver-dominant metastases from thoracic malignancies. J Vasc Interv Radiol 2008;19:299-300.

10 Ahmadzadehfar H, Biersack HJ, Ezziddin S: Radioembolization of liver tumors with yttrium-90 microspheres. Semin Nucl Med 2010;40:105-121.

11 Jemal A, Bray F, Center MM, Ferlay J, Ward E, Forman D: Global cancer statistics. CA Cancer J Clin 2011;61:69-90.

12 Siegel R, Naishadham D, Jemal A: Cancer statistics, 2012. CA Cancer J Clin 2012;62:10-29.

13 Kagohashi K, Satoh H, Ishikawa H, Ohtsuka M, Sekizawa K: Liver metastasis at the time of initial diagnosis of lung cancer. Med Oncol 2003;20:25-28.

14 Disibio G, French SW: Metastatic patterns of cancers: results from a large autopsy study. Arch Pathol Lab Med 2008;132:931-939.

15 Goldstraw P, Crowley J, Chansky K, et al: The IASLC Lung Cancer Staging Project: proposals for the revision of the TNM stage groupings in the forthcoming (seventh) edition of the TNM Classification of malignant tumours. J Thorac Oncol 2007;2:706-714.

16 Fouad H, Metzger T, Tatum C, Robbins K, Martin RC: Hepatic arterial therapy with drug-eluting beads in the management of metastatic bronchogenic carcinoma to the liver: a multi-institutional registry. J Oncol 2012;2012:292131.

17 Salem R, Thurston KG: Radioembolization with 90yttrium microspheres: a state-of-the-art brachytherapy treatment for primary and secondary liver malignancies. Part 2: special topics. J Vasc Interv Radiol 2006;17:1425-1439.

18 SIRflox: http://www.sirflox.com/home (accessed July 19, 2012).

$\checkmark 19$ Salem R, Lewandowski RJ, Sato KT, et al: Technical aspects of radioembolization with 90Y microspheres. Tech Vasc Interv Radiol 2007;10:12-29.

20 Leung TW, Lau WY, Ho SK, et al: Radiation pneumonitis after selective internal radiation treatment with intraarterial 90yttrium-microspheres for inoperable hepatic tumors. Int J Radiat Oncol Biol Phys 1995;33:919-924.

21 Salem R, Parikh P, Atassi B, et al: Incidence of radiation pneumonitis after hepatic intra-arterial radiotherapy with yttrium-90 microspheres assuming uniform lung distribution. Am J Clin Oncol 2008;31:431-438.

-22 Tsujino K, Hirota S, Endo M, et al: Predictive value of dose-volume histogram parameters for predicting radiation pneumonitis after concurrent chemoradiation for lung cancer. Int J Radiat Oncol Biol Phys 2003;55:110-115. 